OPEN ACCESS

Edited by:

Sylvia Anton,

Institut National de la Recherche

Agronomique (INRA), France

Reviewed by:

Christian Wegener,

Universität Würzburg,

Germany

Davide Malagoli,

University of Modena and Reggio

Emilia, Italy

${ }^{*}$ Correspondence:

Stephen B. Shears

shears@niehs.nih.gov

Yoichi Hayakawa

hayakayo@cc.saga-u.ac.jp

Specialty section:

This article was submitted to Invertebrate Physiology, a section of the journal

Frontiers in Physiology

Received: 24 August 2018

Accepted: 21 February 2019

Published: 22 March 2019

Citation:

Shears SB and Hayakawa Y (2019)

Functional Multiplicity of an Insect

Cytokine Family Assists Defense

Against Environmental Stress.

Front. Physiol. 10:222

doi: 10.3389/fphys.2019.00222

\section{Functional Multiplicity of an Insect Cytokine Family Assists Defense Against Environmental Stress}

\author{
Stephen B. Shears ${ }^{1 *}$ and Yoichi Hayakawa ${ }^{2 *}$ \\ 'Inositol Signalling Group, Signal Transduction Laboratory, National Institute of Environmental Health Sciences, National \\ Institutes of Health, Durham, NC, United States, ²Department of Applied Biological Sciences, Saga University, Saga, Japan
}

The widespread distribution of insects over many ecological niches owes much to evolution of multiple mechanisms to defend against environmental stress, especially because their ectothermic nature and small body size render them particularly susceptible to extremes in temperature and water availability. In this review, we will summarize the latest information describing a single, multifunctional cytokine family that is deployed by six orders of insect species to combat a diverse variety of environmental stresses. The originating member of this peptide family was identified in Mythimna (formerly called Pseudaletia) separata armyworm; the cytokine was named growth-blocking peptide (GBP), reflecting its actions in combating parasitic invasion. The peptide's name has been retained, though the list of its regulatory activities has greatly expanded. All members of this family are small peptides, 19-25 amino acid residues, whose major source is fat body. They are now known to regulate embryonic morphogenesis, larval growth rates, feeding activities, immune responses, nutrition, and aging. In this review, we will describe recent developments in our understanding of the mechanisms of action of the GBP family, but we will also highlight remaining gaps in our knowledge.

Keywords: cytokine, growth-blocking peptide (GBP), stress-responsive peptide (SRP), Mthl10, hormesis

\section{INTRODUCTION}

Growth-blocking peptide (GBP) was initially found as a peptidergic factor which blocks JH esterase activation in the hemolymph of early last instar larvae of host Mythimna (formerly called Pseudaletia) separata armyworm upon infection by the parasitic wasp Cotesia kariyai (Hayakawa, 1990). GBP-induced suppression of hemolymph JH esterase is a protective measure that delays larval growth and development (Hayakawa, 1991). Although the mechanism by which M. separata (Ms) GBP suppresses hemolymph JH esterase activation is still unknown, this initial observation led us to focus on its hormone-like function (Hayakawa, 1992). Further characterization of $M s \mathrm{GBP}$ signaling elucidated that it elevates dopamine concentrations in the hemolymph through enhanced expression of tyrosine hydroxylase and DOPA decarboxylase in the integument and the brain (Noguchi et al., 1995, 2003). This up-regulation of gene expression was subsequently attributed to MsGBP-induced activation of phospholipase C (PLC), release of inositol triphosphate (IP3), and the elevation of cytoplasmic $\mathrm{Ca}^{2+}$ concentrations (Ninomiya and Hayakawa, 2007; Ninomiya et al., 2008). Although the relationship between 
dopamine elevation and $\mathrm{JH}$ esterase repression has not been yet clarified, both events have negative impact on the growth rates of insect larvae (Noguchi et al., 1995).

In the years since the discovery of $M s \mathrm{GBP}$, over $10 \mathrm{GBP}$ orthologous peptides have been found in several lepidopteran species (Hayakawa, 1995, 2006). They all consist of 23-25 amino acids, and they share more than 70\% sequence identity, yet they show diverse functions: paralysis induction, plasmatocyte spreading, and cardioacceleration. However, to date, the only known receptor for a GBP is that identified in DrosophilaMthl10 (see below). MsGBP itself was demonstrated to have this multifunctionality (Strand et al., 2000). Subsequent studies established further functions of the GBP family such as cell growth activator, early morphogenetic mediator, and humoral immune mediator (Ohnishi et al., 2001; Tsuzuki et al., 2005, 2012). Nevertheless, as is common practice, this cytokine family is still named after its originating function as a growth-blocking peptide (Hayakawa, 2006).

\section{NONLEPIDOPTERAN GBP}

Many GBP orthologous peptides had been reported in Lepidoptera, but it was not until 2012 that the first nonlepidopteran GBP was discovered (Tsuzuki et al., 2012). To identify their primary structures, hemolymph peptides that induce cell growth and plasmatocyte spreading activities were purified from Tenebrionid and bluebottle fly larvae (Matsumoto et al., 2012; Tsuzuki et al., 2012). The functional orthologs identified by these studies comprised 19-24 amino acids, and subsequent homology searches expanded the presence of GBP-like peptides to five orders. Comparisons of these peptides enabled us to extract the consensus motif C-x(2)-G-x(4,6)-G-x(1,2)-C-[KR] (Matsumoto et al., 2012). More recently, this motif has been found in Locusta migratoria and Schistocerca gregaria GBPs (Duressa et al., 2015). Here, we describe the phylogenetic relationship derived from precursor protein sequences of all known members of the GBP family and GBP orthologs which were identified by homology searches (Figure 1). It is interesting that the GBP motif shares a significant similarity with the portion of the mammalian epidermal growth factor (EGF) motif (Figure 2) that forms the C-terminal region, in which Arg41 and Leu47 have been reported to be crucial for binding to the EGF receptor (Ogiso et al., 2002). NMR analysis demonstrated that the GBP motif core structure (residues 7-22) is predicted to show an EGF-like fold stabilized by a disulfide bond and a short B-hairpin turn (Aizawa et al., 1999). This characteristic tertiary structure has been reported to be common in lepidopteran GBP orthologs which had been previously referred as to "ENF-peptide" that was named after the consensus N-terminal amino acid sequence (Volkman et al., 1999; Yu et al., 1999).

Although several other insect cytokines, such as Spätzle (DeLotto and DeLotto, 1998; Weber et al., 2003), Unpaired (Zeidler et al., 1999; Karsten et al., 2002; Yang et al., 2015), and Eiger (Moreno et al., 2002), have been reported, most of them were identified by searching for Drosophila orthologs of human cytokines. Therefore, GBP is unique in that following its original discovery in the armyworm, and it has since been identified in many other insect species, but no human ortholog has been found yet (Vanha-Aho et al., 2016). It is therefore particularly intriguing that Drosophila melanogaster (Dm) GBP exhibits some sequence similarity with human defensin $\mathrm{BD} 2$, a member of the immunomodulatory $ß$-defensin family, that can also regulate cell proliferation; BD2 is small, cationic peptides produced by specific proteolytic processing just like DmGBP (Shafee et al., 2017). Furthermore, both the GBP and defensin families recruit the inositol phosphate (IP) $/ \mathrm{Ca}^{2+}$ signaling cascade to serve their biological actions in common (Niyonsaba et al., 2007; Ninomiya et al., 2008; Zhou et al., 2012; Tsuzuki et al., 2014).

\section{DROSOPHILA GBP}

Following on from the identification of $D m \mathrm{GBP}$, three major developments have been made concerning its functions and signaling mechanisms as follows. First, $D m G B P$ was demonstrated to elevate anti-microbial peptide (AMP) expression independently of the canonical receptors that at that time were known to be associated with the inflammatory pathways mediated by Toll- and IMD-dependent pathways (Tsuzuki et al., 2012). Instead, the adaptor protein IMD is recruited to an activated $D m G B P$ receptor which thereby activates JNK. This signaling pathway stimulates expression of a unique set of AMP genes, mainly Mechnikowin and Diptericin. The DmGBP-dependent $A M P$ expression occurs not only in larvae infected with pathogens but also in larvae exposed to noninfectious stress such as high/low temperatures or mechanical perturbation; thus, GBP has more general roles in maintaining insect homeostasis.

Second, it was demonstrated that $D m G B P$ activates an $\mathrm{IP} / \mathrm{Ca}^{2+}$ signaling cascade that dictates the timing and the intensity of the separate cellular and humoral components of the innate immune response which, moreover, are reciprocally regulated (Tsuzuki et al., 2014). DmGBP protects against pathogens by activating cellular defense program (phagocytosis and encapsulation), while inhibiting humoral pathways (production and release of AMPs), through an $\mathrm{IP} / \mathrm{Ca}^{2+}$ signaling-mediated activation of a receptor-regulated kinase cascade (the PVR/ERK pathway).

Third, by screening a dsRNA library that targets genes encoding membrane proteins, the $D m G B P$ receptor has been determined to be the G-protein-coupled receptor Methuselahlike 10 (Mthl10) (Sung et al., 2017). Knockdown of Mthl10 by RNAi resulted in increased mortality upon bacterial infection and impaired adaptation to an environmental stress such as cold temperature.

It was recently reported that $D m \mathrm{GBP}$ regulates the release of insulin-like peptides (ILPs) from the brain depending on nutrient levels in the hemolymph through target of rapamycin (TOR) in Drosophila (Koyama and Mirth, 2016). Thus, it was investigated if the GBP elicited those effects by acting through Mthl10. Mthl10 was found to be expressed in ILP-producing cells of the brain and Mthl10 knockdown decreased ILP expression (Sung et al., 2017). Mthl10 knockdown was also demonstrated to be associated with 


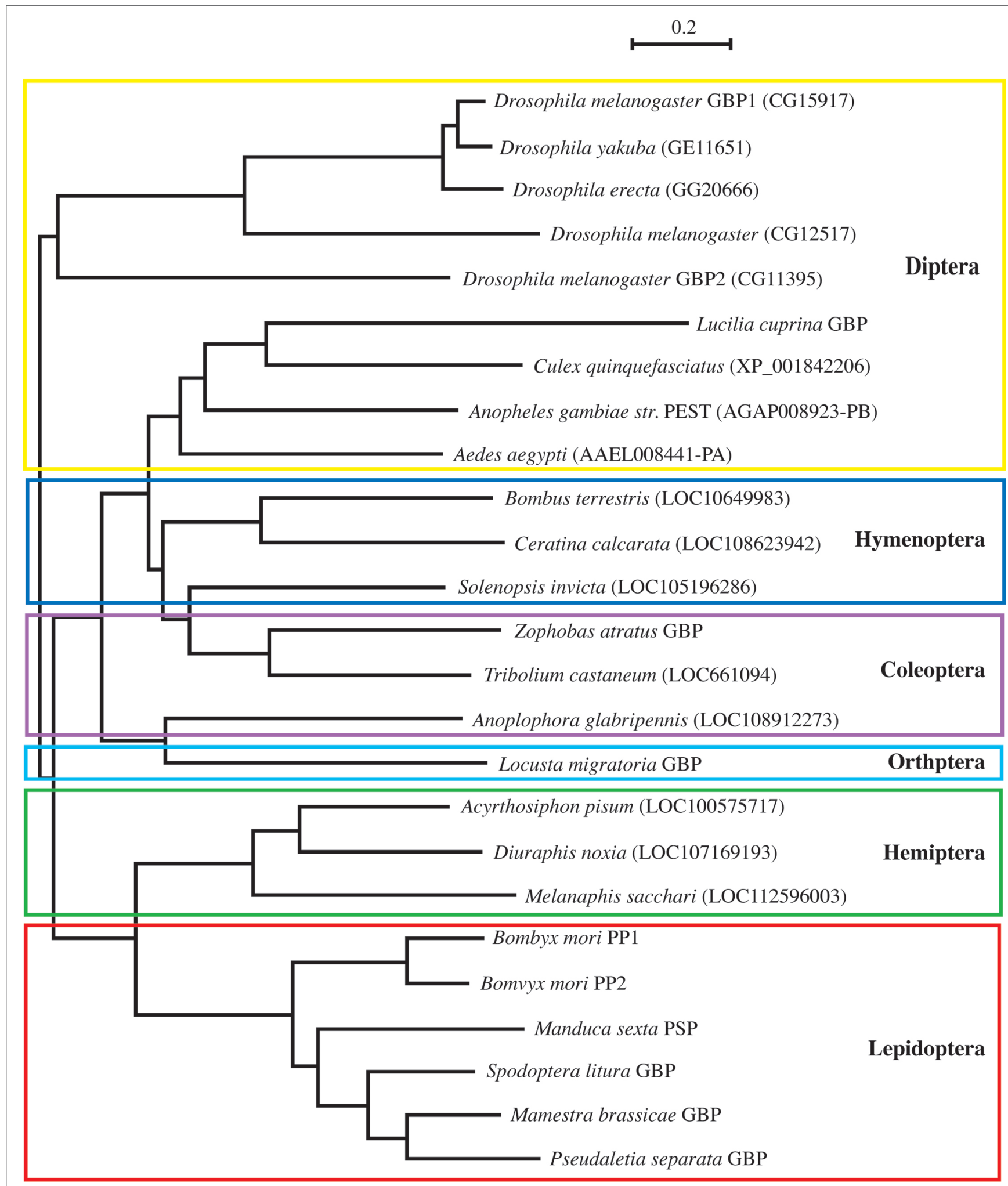

FIGURE 1 | A phylogenetic tree derived from precursor polypeptide sequences of GBP and GBP-like gene family spanning six orders of insects by using the neighbor-joining method with protein-Poisson distances (Saitou and Nei, 1987). The following sequences were identified on database of the NCBI/Blast: Diuraphis noxia LOC107169193 (XP_015374346.1), Melanaphis sacchari LOC112596003 (XP_025197225.1), Solenopsis invicta LOC105196286 (XP_011160410), Ceratina calcarata LOC108623942 (XP_017878339), and Anoplophora glabripennis LOC108912273 (XP_018572983). Locusta migratoria GBP was reported by Durressa et al. (Duressa et al., 2015). Other peptide sequences are in the prior report (Matsumoto et al., 2012). PP: paralytic peptide and PSP: plasmatocyte spreading peptide. Scale bar means a number of amino acid substitution per site. 


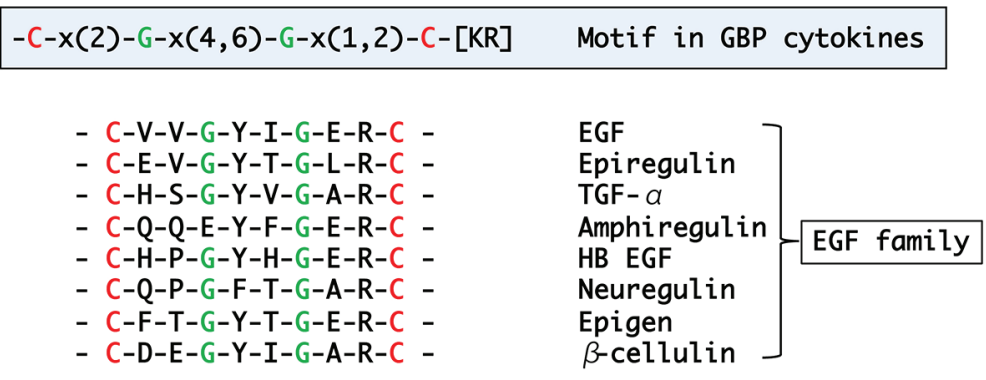

FIGURE 2 | A motif found in the active peptide regions of GBP/GBP-like genes and alignment of mammalian EGF peptide family.

increased longevity of flies, while $D m G B P$ overexpression shortened lifespans. Furthermore, the GBP-induced shorterlived phenotype was not observed in a strain with simultaneous knockdown of Mthl10. These observations provided solid evidence that Mthl10-mediated integration of various immunological and metabolic properties of $D m \mathrm{GBP}$ is essential to maintain health and homeostasis that are critical for normal lifespan in insects.

\section{GBP SIGNALING AND ITS REGULATION}

In mammals, the cytokine TNF triggers the production of proinflammatory cytokines such as IL-1ß and IL-6 (Cunha et al., 1991; Lorenzetti et al., 2002). Furthermore, IL-6 expression has been demonstrated to be induced by IL- $1 ß$ in epithelial cells (Moon et al., 2000; Khan et al., 2014). Another insect cytokine, stress-responsive peptide (SRP), was recently identified; its expression is enhanced by $M s G B P$ in the armyworm (Yamaguchi et al., 2012; Matsumura et al., 2018). Physiological functions of SRP are similar to those of $M s \mathrm{GBP}$. For example, both $M s \mathrm{GBP}$ and SRP showed larval growth retardation when they are injected into early last instar larvae. Although MsGBP elicits a slightly stronger growth inhibitory effect than SRP, co-injection of both peptides has a greater effect than that due to MsGBP alone (Yamaguchi et al., 2012; Matsumura et al., 2018). The negative impact on larval growth seems to be mainly due to the MsGBP and/or SRP-induced decrease in larval feeding activities: co-injection of both cytokines caused a slightly more severe reduction in appetite than injection of each individual factor alone. Similar effects by cytokines have been reported in mouse IL-1ß and IL-6: both cytokines synergistically enhanced STAT3/NF-кBdependent gene expression in the mouse liver during the acute inflammation phase (Goldstein et al., 2017). It might be worth investigating the functional parallelism between $M s$ GBP-SRP and IL-1ß_IL-6 to clarify evolutional feature of cytokine functions. Furthermore, it was demonstrated that $M s \mathrm{GBP}$ does not elevate $S R P$ expression when injected with SRP into the armyworm larvae (Matsumura et al., 2018), indicating that MsGBP cannot activate $S R P$ expression as long as SRP is present in the hemolymph above a threshold concentration. This might be analogous to the fact that an excessive immune response, through strong stress, stimulates a negative feedback mechanism in mammals, which protects the organism from an overproduction of proinflammatory cytokines (Elenkov and Chrousos, 2002).

Another mode of GBP signaling regulation is the control of its hemolymph concentrations by GBP-binding protein (GBP-BP) that functions as a scavenger of $M s \mathrm{GBP}$ in the armyworm (Matsumoto et al., 2003). As mentioned above, DmGBP initially tends to prioritize neutralization of an invading pathogen by activating cellular defense reactions (spreading, phagocytosis, and encapsulation). $M s G B P$ regulates not only immune active plasmatocytes and granulocytes in Lepidoptera (Lavine and Strand, 2002), but also another hemocyte class, the oenocytoids. The latter cells possess densely packed GBP-BP molecules, which are released by $M s \mathrm{GBP}$-induced cell lysis that occurs after the cellular immune responses of plasmatocytes (Matsumoto et al., 2003). Therefore, MsGBP has temporally dependent actions, first to stimulate the immune cells and afterwards to silence its own action by releasing GBP-BP through specific hemolysis of oenocytoids. Although an equivalent GBP-BP has not been identified in Drosophila, orthologous genes and proteins have been identified in several Lepidoptera such as Manduca sexta (Chevignon et al., 2015), Bombyx mori (Hu et al., 2006; Sasibhushan et al., 2013), Spodoptera exigua (Park and Kim, 2012), Spodoptera frugiperda (Barat-Houari et al., 2006), and Helicoverpa armigera (Shelby and Popham, 2009). Bacterial and viral infection has been reported to enhance expression of GBP-BP genes in the hemocytes of some lepidopteran larvae, which supports the proposed immunological functional role of this protein. Moreover, expression of GBP-BP is dependent on the dependent stage of the insect and is enhanced by 20-hydroxyecdysone (20E), which together suggests that there are other consequences for the interaction of GBP with GBP-BP (Zhuo et al., 2018). For example, GBP and its binding protein may exert metabolic regulation during metamorphosis; down-regulation of metabolic levels by clearance of hemolymph GBP by GBP-BP would help the normal process of metamorphosis because it is well known that insects become inactive during metamorphosis. Furthermore, it has been shown that there are sharp GBP peaks in the hemolymph during each larval molt (Ohnishi et al., 1995). It is possible that GBP-BP contributes toward purging hemolymph GBP after the shut-off of its gene expression, which could make the sharp GBP peaks during molt periods. 


\section{FUTURE INVESTIGATIONS OF GBP SIGNALING}

There remain many important questions regarding GBP multifunctionality and their regulation. For example, it has been demonstrated that GBP serves its immunological and metabolic functions as described above. Furthermore, GBP functions as a cell growth factor (Hayakawa and Ohnishi, 1998; Matsumoto et al., 2012). It has been reported that MsGBP acts as a bipolar growth regulator: high concentrations (over several $10 \mathrm{pmol} / \mathrm{ml}$ ) suppress larval growth but low concentrations (several $\mathrm{pmol} / \mathrm{ml}$ ) enhance larval growth and cell proliferation (Hayakawa and Ohnishi, 1998). In fact, several $\mathrm{pmol} / \mathrm{ml}$ of $M s \mathrm{GBP}$ enhances proliferation of human keratinocytes and of SF-9 insect cells in a manner similar to mammalian EGF (Hayakawa and Ohnishi, 1998). It is not yet known if Mthl10 contributes to DmGBP-dependent cell proliferation. Indeed, based on the prior results obtained by structural (Aizawa et al., 1999) and kinetic studies (Hayakawa and Ohnishi, 1998; Ohnishi et al., 2001), it is reasonable to expect that stimulation of cell growth by GBP requires another type of the receptor similar to the EGF family of receptor tyrosine kinases. The speculation that GBP could activate multiple receptor types has arisen from the demonstration that different minimal peptide sequences of $M s \mathrm{GBP}$ are required for cell growth and cellular immune activities: residues 2-23 in GBP are required for the former activity and 1-22 in GBP for the latter (Aizawa et al., 2001).

When DmGBP (CG15917) was first identified, four other Drosophila genes encoding the proGBP-like peptide were also found: CG11395, CG12517, CG14069, and CG17244. Koyama and Mirth recently found that the CG11397 gene product regulated the release of ILPs from the brain in the similar manner of GBP (CG15917) and they named CG15917 and CG11397 for GBP1 and GBP2, respectively (Koyama and Mirth, 2016). The role of these two DmGBPs in metabolic regulation has been demonstrated, but it has not yet been checked whether GBP2 also shares similar immune regulatory functions with GBP1. Moreover, it remains to be seen if the other candidate

\section{REFERENCES}

Aizawa, T., Fujitani, N., Hayakawa, Y., Ohnishi, A., Ohkubo, T., Kumaki, Y., et al. (1999). Solution structure of an insect growth factor, growth-blocking peptide. J. Biol. Chem. 274, 1887-1890. doi: 10.1074/jbc.274.4.1887

Aizawa, T., Hayakawa, Y., Ohnishi, A., Fujitani, N., Clark, K. D., Strand, M. R., et al. (2001). Structure and activity of the insect cytokine growth-blocking peptide. Essential regions for mitogenic and hemocytestimulating activities are separate. J. Biol. Chem. 276, 31813-31818. doi: 10.1074/jbc.M105251200

Barat-Houari, M., Hilliou, F., Jousset, F. X., Sofer, L., Deleury, E., Rocher, J., et al. (2006). Gene expression profiling of Spodoptera frugiperda hemocytes and fat body using cDNA microarray reveals polydnavirus-associated variations in lepidopteran host genes transcript levels. BMC Genomics 7:160. doi: 10.1186/1471-2164-7-160

Chevignon, G., Cambier, S., Da Silva, C., Poulain, J., Drezen, J. M., Huguet, E., et al. (2015). Transcriptomic response of Manduca sexta immune tissues to parasitization by the bracovirus associated wasp Cotesia congregata. Insect Biochem. Mol. Biol. 62, 86-99. doi: 10.1016/j.ibmb.2014.12.008 genes described above (CG12517, CG14069, and CG17244) will turn out to expand the functionality of the DmGBP family.

\section{CONCLUSIONS}

Although the multiple functionalities of $M s G B P$ and $D m G B P$ have been clearly demonstrated, it remains unclear to what extent the GBP-signaling pathways and functionalities are conserved in other insects. For example, it is not yet known if Mthl10 orthologous gene occurs in the armyworm. Moreover, SRP and GBP-BP have been examined only in the armyworm. It will be important to identify all these essential components for GBP-signaling function and regulation in broad insect species, so as to identify species-, development-, and stage-specific expression of such components. Elucidating commonality and difference of such GBP-associated factors in insects may hint at the conservation of some of these important homeostatic mechanisms in mammals.

\section{AUTHOR CONTRIBUTIONS}

All authors listed have made a substantial, direct and intellectual contribution to the work, and approved it for publication.

\section{FUNDING}

This research was supported by the Intramural Research Program of the NIH, National Institutes of Environmental Health Sciences (SS) and by a Grant-in-Aid for Scientific Research (A) (Grant number: 16H0259) from JSPS (YH).

\section{ACKNOWLEDGMENTS}

We appreciate the contribution of Dr. Hitoshi Matsumoto (Saga University) in drawing the phylogenetic tree.

Cunha, F. Q., Lorenzetti, B. B., Poole, S., and Ferreira, S. H. (1991). Interleukin-8 as a mediator of sympathetic pain. Br. J. Pharmacol. 104, 765-767. doi: 10.1111/j.1476-5381.1991.tb12502.x

DeLotto, Y., and DeLotto, R. (1998). Proteolytic processing of the Drosophila Spatzle protein by easter generates a dimeric NGF-like molecule with ventralising activity. Mech. Dev. 72, 141-148. doi: 10.1016/S0925-4773(98)00024-0

Duressa, T. F., Boonen, K., Hayakawa, Y., and Huybrechts, R. (2015). Identification and functional characterization of a novel locust peptide belonging to the family of insect growth blocking peptides. Peptides 74, 23-32. doi: 10.1016/j. peptides.2015.09.011

Elenkov, I. J., and Chrousos, G. P. (2002). Stress hormones, proinflammatory and antiinflammatory cytokines, and autoimmunity. Ann. N. Y. Acad. Sci. 966, 290-303. doi: 10.1111/j.1749-6632.2002.tb04229.x

Goldstein, I., Paakinaho, V., Baek, S., Sung, M. H., and Hager, G. L. (2017). Synergistic gene expression during the acute phase response is characterized by transcription factor assisted loading. Nat. Commun. 8:1849. doi: 10.1038/ s41467-017-02055-5

Hayakawa, Y. (1990). Juvenile hormone esterase activity repressive factor in the plasma of parasitized insect larvae. J. Biol. Chem. 265, 10813-10816. 
Hayakawa, Y. (1991). Structure of a growth-blocking peptide present in parasitized insect hemolymph. J. Biol. Chem. 266, 7981-7984.

Hayakawa, Y. (1992). A putative new juvenile peptide hormone in lepidopteran insects. Biochem. Biophys. Res. Commun. 185, 1141-1147. doi: 10.1016/0006-291X(92)91745-C

Hayakawa, Y. (1995). Growth-blocking peptide: an insect biogenic peptide that prevents the onset of metamorphosis. J. Insect Pysiology 41, 1-6.

Hayakawa, Y. (2006). Insect cytokine growth-blocking peptide (GBP) regulates insect development. Appl. Entomol. Zool. 41, 545-554. doi: 10.1303/aez.2006.545

Hayakawa, Y., and Ohnishi, A. (1998). Cell growth activity of growth-blocking peptide. Biochem. Biophys. Res. Commun. 250, 194-199. doi: 10.1006/ bbrc. 1998.8959

Hu, Z. G., Chen, K. P., Yao, Q., Gao, G. T., Xu, J. P., and Chen, H. Q. (2006). Cloning and characterization of Bombyx mori PP-BP a gene induced by viral infection. Yi Chuan Xue Bao. Acta Gene. Sin. 33, 975-983. doi: 10.1016/ S0379-4172(06)60132-7

Karsten, P., Hader, S., and Zeidler, M. P. (2002). Cloning and expression of Drosophila SOCS36E and its potential regulation by the JAK/STAT pathway. Mech. Dev. 117, 343-346. doi: 10.1016/S0925-4773(02)00216-2

Khan, Y. M., Kirkham, P., Barnes, P. J., and Adcock, I. M. (2014). Brd4 is essential for IL-1beta-induced inflammation in human airway epithelial cells. PLoS One 9:e95051. doi: 10.1371/journal.pone.0095051

Koyama, T., and Mirth, C. K. (2016). Growth-blocking peptides as nutritionsensitive signals for insulin secretion and body size regulation. PLoS Biol. 14:e1002392. doi: 10.1371/journal.pbio.1002551

Lavine, M. D., and Strand, M. R. (2002). Insect hemocytes and their role in immunity. Insect Biochem. Mol. Biol. 32, 1295-1309. doi: 10.1016/ S0965-1748(02)00092-9

Lorenzetti, B. B., Veiga, F. H., Canetti, C. A., Poole, S., Cunha, F. Q., and Ferreira, S. H. (2002). Cytokine-induced neutrophil chemoattractant 1 (CINC-1) mediates the sympathetic component of inflammatory mechanical hypersensitivitiy in rats. Eur. Cytokine Netw. 13, 456-461.

Matsumoto, H., Tsuzuki, S., Date-Ito, A., Ohnishi, A., and Hayakawa, Y. (2012). Characteristics common to a cytokine family spanning five orders of insects. Insect Biochem. Mol. Biol. 42, 446-454. doi: 10.1016/j.ibmb.2012.03.001

Matsumoto, Y., Oda, Y., Uryu, M., and Hayakawa, Y. (2003). Insect cytokine growth-blocking peptide triggers a termination system of cellular immunity by inducing its binding protein. J. Biol. Chem. 278, 38579-38585. doi: 10.1074/jbc.M305986200

Matsumura, T., Nakano, F., Matsumoto, H., Uryu, O., and Hayakawa, Y. (2018). Identification of a cytokine combination that protects insects from stress. Insect Biochem. Mol. Biol. 97, 19-30. doi: 10.1016/j.ibmb.2018.04.002

Moon, M. R., Parikh, A. A., Pritts, T. A., Kane, C., Fischer, J. E., Salzman, A. L., et al. (2000). Interleukin-1beta induces complement component $\mathrm{C} 3$ and IL-6 production at the basolateral and apical membranes in a human intestinal epithelial cell line. Shock 13, 374-378. doi: 10.1097/00024382-200005000-00005

Moreno, E., Yan, M., and Basler, K. (2002). Evolution of TNF signaling mechanisms: JNK-dependent apoptosis triggered by Eiger, the Drosophila homolog of the TNF superfamily. Curr. Biol. 12, 1263-1268. doi: 10.1016/ S0960-9822(02)00954-5

Ninomiya, Y., and Hayakawa, Y. (2007). Insect cytokine, growth-blocking peptide, is a primary regulator of melanin-synthesis enzymes in armyworm larval cuticle. FEBS J. 274, 1768-1777. doi: 10.1111/j.1742-4658.2007.05724.x

Ninomiya, Y., Kurakake, M., Oda, Y., Tsuzuki, S., and Hayakawa, Y. (2008). Insect cytokine growth-blocking peptide signaling cascades regulate two separate groups of target genes. FEBS J. 275, 894-902. doi: 10.1111/j. 1742-4658.2008.06252.x

Niyonsaba, F., Ushio, H., Nakano, N., Ng, W., Sayama, K., Hashimoto, K., et al. (2007). Antimicrobial peptides human beta-defensins stimulate epidermal keratinocyte migration, proliferation and production of proinflammatory cytokines and chemokines. J. Invest. Dermatol. 127, 594-604. doi: 10.1038/ sj.jid.5700599

Noguchi, H., Hayakawa, Y., and Downer, R. (1995). Elevation of dopamine levels in parasitized insect larvae. Insect Biochem. Mol. Biol. 25, 197-201. doi: 10.1016/0965-1748(94)00054-L

Noguchi, H., Tsuzuki, S., Tanaka, K., Matsumoto, H., Hiruma, K., and Hayakawa, Y. (2003). Isolation and characterization of a dopa decarboxylase cDNA and the induction of its expression by an insect cytokine, growth-blocking peptide in
Pseudaletia separata. Insect Biochem. Mol. Biol. 33, 209-217. doi: 10.1016/ S0965-1748(02)00192-3

Ogiso, H., Ishitani, R., Nureki, O., Fukai, S., Yamanaka, M., Kim, J. H., et al. (2002). Crystal structure of the complex of human epidermal growth factor and receptor extracellular domains. Cell 110, 775-787. doi: 10.1016/ S0092-8674(02)00963-7

Ohnishi, A., Hayakawa, Y., Matsuda, Y., Kwon, K. W., Takahashi, T. A., and Sekiguchi, S. (1995). Growth-blocking peptide titer during larval development of parasitized and cold-stressed armyworm. Insect Biochem. Mol. Biol. 25, 1121-1127. doi: 10.1016/0965-1748(95)00054-2

Ohnishi, A., Oda, Y., and Hayakawa, Y. (2001). Characterization of receptors of insect cytokine, growth-blocking peptide, in human keratinocyte and insect Sf9 cells. J. Biol. Chem. 276, 37974-37979. doi: 10.1074/jbc.M104856200

Park, J. A., and Kim, Y. (2012). Toll recognition signal activates oenocytoid cell lysis via a crosstalk between plasmatocyte-spreading peptide and eicosanoids in response to a fungal infection. Cell. Immunol. 279, 117-123. doi: 10.1016/j. cellimm.2012.11.005

Saitou, N., and Nei, M. (1987). The neighbor-joining method: a new method for reconstructing phylogenetic trees. Mol. Biol. Evol. 4, 406-425. doi: 10.1093/ oxfordjournals.molbev.a040454

Sasibhushan, S., C. G. P. R., and Ponnuvel, K. M. (2013). Genome wide microarray based expression profiles during early embryogenesis in diapause induced and non-diapause eggs of polyvoltine silkworm Bombyx mori. Genomics 102, 379-387. doi: 10.1016/j.ygeno.2013.07.007

Shafee, T. M., Lay, F. T., Phan, T. K., Anderson, M. A., and Hulett, M. D. (2017). Convergent evolution of defensin sequence, structure and function. Cell. Mol. Life Sci. 74, 663-682. doi: 10.1007/s00018-016-2344-5

Shelby, K. S., and Popham, H. J. (2009). Analysis of ESTs generated from immune-stimulated hemocytes of larval Heliothis virescens. J. Invertebr. Pathol. 101, 86-95. doi: 10.1016/j.jip.2009.05.002

Strand, M. R., Hayakawa, Y., and Clark, K. D. (2000). Plasmatocyte spreading peptide (PSP1) and growth blocking peptide (GBP) are multifunctional homologs. J. Insect Pysiol. 46, 817-824.

Sung, E. J., Ryuda, M., Matsumoto, H., Uryu, O., Ochiai, M., Cook, M. E., et al. (2017). Cytokine signaling through Drosophila Mthl10 ties lifespan to environmental stress. Proc. Natl. Acad. Sci. U. S. A. 114, 13786-13791. doi: $10.1073 /$ pnas. 1712453115

Tsuzuki, S., Matsumoto, H., Furihata, S., Ryuda, M., Tanaka, H., Sung, E. J., et al. (2014). Switching between humoral and cellular immune responses in Drosophila is guided by the cytokine GBP. Nat. Commun. 5:4628. doi: $10.1038 /$ ncomms5628

Tsuzuki, S., Ochiai, M., Matsumoto, H., Kurata, S., Ohnishi, A., and Hayakawa, Y. (2012). Drosophila growth-blocking peptide-like factor mediates acute immune reactions during infectious and non-infectious stress. Sci. Rep. 2:210. doi: 10.1038/ srep00210

Tsuzuki, S., Sekiguchi, S., Kamimura, M., Kiuchi, M., and Hayakawa, Y. (2005). A cytokine secreted from the suboesophageal body is essential for morphogenesis of the insect head. Mech. Dev. 122, 189-197. doi: 10.1016/j. mod.2004.10.005

Vanha-Aho, L. M., Valanne, S., and Ramet, M. (2016). Cytokines in Drosophila immunity. Immunol. Lett. 170, 42-51. doi: 10.1016/j.imlet.2015.12.005

Volkman, B. F., Anderson, M. E., Clark, K. D., Hayakawa, Y., Strand, M. R., and Markley, J. L. (1999). Structure of the insect cytokine peptide plasmatocytespreading peptide 1 from Pseudoplusia includens. J. Biol. Chem. 274, 4493-4496. doi: 10.1074/jbc.274.8.4493

Weber, A. N., Tauszig-Delamasure, S., Hoffmann, J. A., Lelievre, E., Gascan, H., Ray, K. P., et al. (2003). Binding of the Drosophila cytokine Spatzle to Toll is direct and establishes signaling. Nat. Immunol. 4, 794-800. doi: $10.1038 /$ ni 955

Yamaguchi, K., Matsumoto, H., Ochiai, M., Tsuzuki, S., and Hayakawa, Y. (2012). Enhanced expression of stress-responsive cytokine-like gene retards insect larval growth. Insect Biochem. Mol. Biol. 42, 183-192. doi: 10.1016/j. ibmb.2011.11.009

Yang, H., Kronhamn, J., Ekstrom, J. O., Korkut, G. G., and Hultmark, D. (2015). JAK/STAT signaling in Drosophila muscles controls the cellular immune response against parasitoid infection. EMBO Rep. 16, 1664-1672. doi: 10.15252/embr.201540277

Yu, X. Q., Prakash, O., and Kanost, M. (1999). Structure of a paralytic peptide from an insect, Manduca sexta. J. Pept. Res. 54, 256-261. 
Zeidler, M. P., Perrimon, N., and Strutt, D. I. (1999). Polarity determination in the Drosophila eye: a novel role for unpaired and JAK/STAT signaling. Genes Dev. 13, 1342-1353. doi: 10.1101/gad.13.10.1342

Zhou, Y., Wu, S., Wang, H., Hayakawa, Y., Bird, G. S., and Shears, S. B. (2012). Activation of PLC by an endogenous cytokine (GBP) in Drosophila S3 cells and its application as a model for studying inositol phosphate signalling through ITPK1. Biochem. J. 448, 273-283. doi: 10.1042/BJ20120730

Zhuo, X. R., Chen, L., Wang, G. J., Liu, X. S., Wang, Y. F., Liu, K., et al. (2018). 20-Hydroxyecdysone promotes release of GBP-binding protein from oenocytoids to suppress hemocytic encapsulation. Insect Biochem. Mol. Biol. 92, 53-64. doi: 10.1016/j.ibmb.2017.11.006
Conflict of Interest Statement: The authors declare that the research was conducted in the absence of any commercial or financial relationships that could be construed as a potential conflict of interest.

Copyright $\odot 2019$ Shears and Hayakawa. This is an open-access article distributed under the terms of the Creative Commons Attribution License (CC BY). The use, distribution or reproduction in other forums is permitted, provided the original author(s) and the copyright owner(s) are credited and that the original publication in this journal is cited, in accordance with accepted academic practice. No use, distribution or reproduction is permitted which does not comply with these terms. 